\title{
The research on the design concept of the campus planning of national Taiwan university-Taking National Kaohsiung First University of science and technology as an example
}

\author{
Shuai Yang \\ School of Art and Design, Guangdong University of Technology Guangzhou 510000, China \\ Mr.yangshuai@qq.com
}

Keywords: Campus; Planning; Environmental Design.

\begin{abstract}
This paper is about the development process of higher education in Taiwan, compared the four different age characteristics of campus planning in Taiwan .And it explained the concept of the formation of Taiwan's campus planning, development. It summed up the characteristics of the design in Taiwan today, through the analysis of campus planning of Kaohsiung First University of Science and Technology.
\end{abstract}

\section{Introduction}

Taiwan Kaohsiung First University of science and technology is the school with the Kaohsiung Institute, Site is located in Taiwan District of Kaohsiung city Nantz, Come out in front in southern Taiwan Technology University. In the campus planning within the scope of Zhongshan expressway, from the south to the north across the campus. In the early stages of planning, will be campus design into two campuses, and the functional areas of the classification settings, East Campus for teaching and administrative district, West Campus for students living area. In the planning and design of campus, embodies the following design concept.

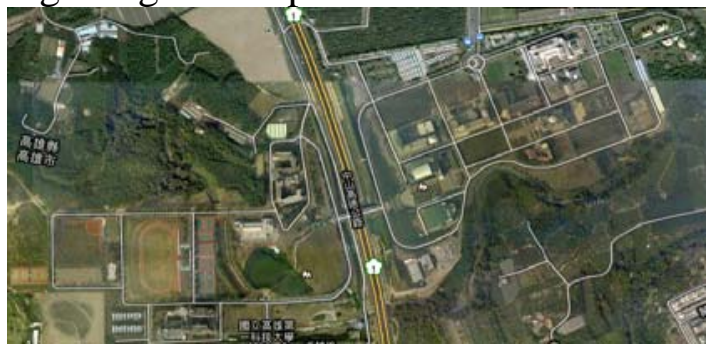

Fig. 1 from the www.google.com

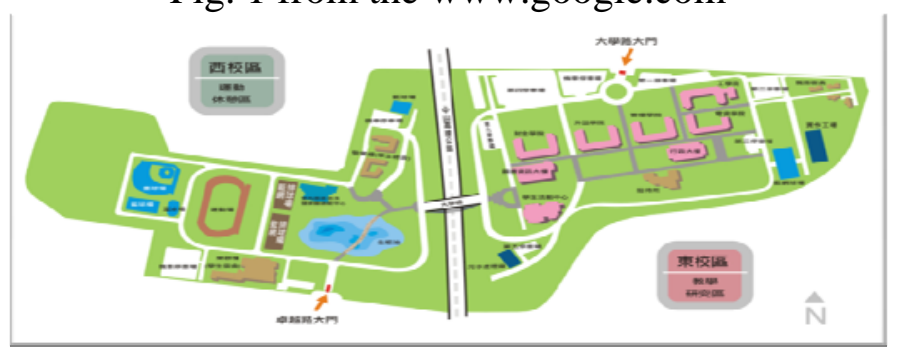

Fig. 2 Campus plan from the www.nkfust.edu.tw

\section{The overall layout of multi campus}

In the design of the east campus, it uses linear programming. In order to spindle center, Institute, Institute of finance, foreign language school, library, administration building 7 buildings. Independent Institute of Architecture, ensures that the teachers in relation to scientific research spaces and the students are having class quiet spaces, with the linear layout of the teaching area, each part is relatively independent. Easy to contact, suitable for synchronous development. In the east side of the east campus, the establishment of production study research area, in this region, which is 
relatively noisy machines and other test equipment. It is due to the linear distribution, let the research study area away from the normal teaching place, and not on the students' learning effect.
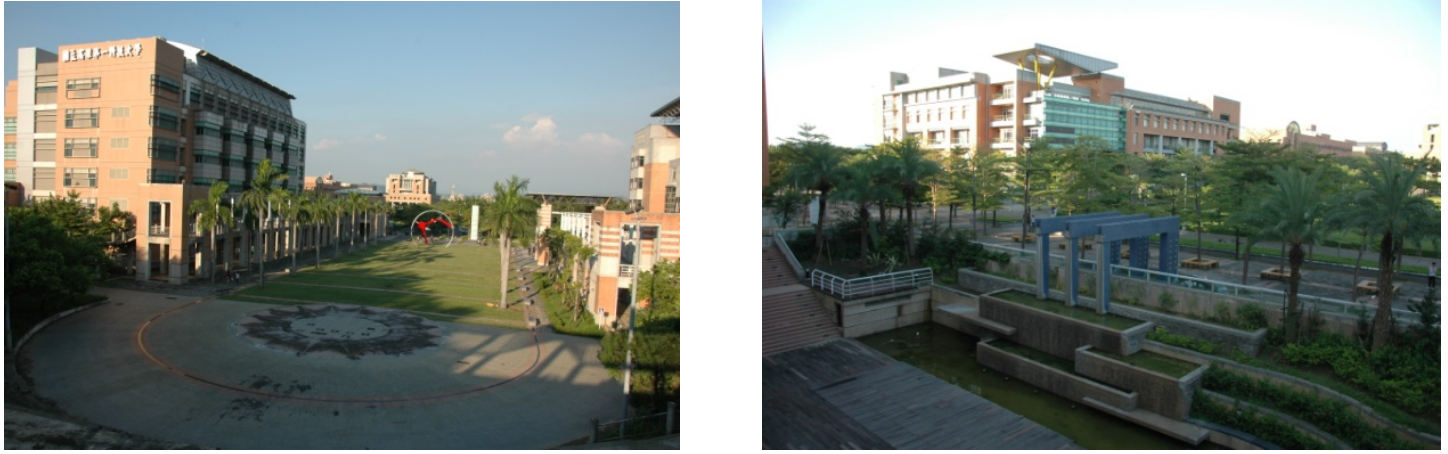

Fig. 3-4. In a corner of the East Campus of spindle

West Campus for students living area, including the girls' dormitory, dormitory, dining room, the ecological pond, swimming pool, basketball courts, tennis courts, baseball, track and field and so on. The West Campus of the design concept spread to the central core, the ecological pond as the core, other regional distribution planning. The design of green space as the core to the scattered around, equipment is perfect, convenient for students in leisure activities. In the center of the lake, landscaping and road construction of a system. Along the lake walk loop, in order to meet the traffic function at the same time, combined with the terrain character line drawing of two kinds of landscape. The curve of Luqiao, considered the "hydrophilic characteristics of people born with the". The hydrophilic step, people and water can be direct contact and exchange, is a rational expression. The embankment with natural and smooth curve formed by water, lake water and grass, split stone processing technique, its theme is to create the dialog between man and nature to the richness of plant communities of natural landscape and natural, romantic display. Jinghu stroll, walking King vary along the lake, view of the beautiful natural landscape, let a person feel fresh. Is a good place for students to relax, sit side by side and talk intimately.



Fig. 5 West campus, the center of the lake

\section{Excellent green space design}

Campus greening as one of the important factors of the campus environment, is not only an important part of the campus material environment, also an important symbol of the civilization construction of the campus. A lot of green plants can regulate oxygen and carbon dioxide in the air balance effect, can make the campus to keep the air fresh. For the colleges and universities, students and teachers engaged in the high strength, high load of mental work, good air quality will provide a fresh and healthy working and learning environment for teachers and students. The plant has the adsorption of harmful substances, dust filter in the air, so as to reduce the adverse effects of external factors on the campus environment. At the same time, it can attenuate the noise. Kaohsiung First University of science and technology, the extensive use of the campus green vegetation, coverage rate reached more than $80 \%$. 

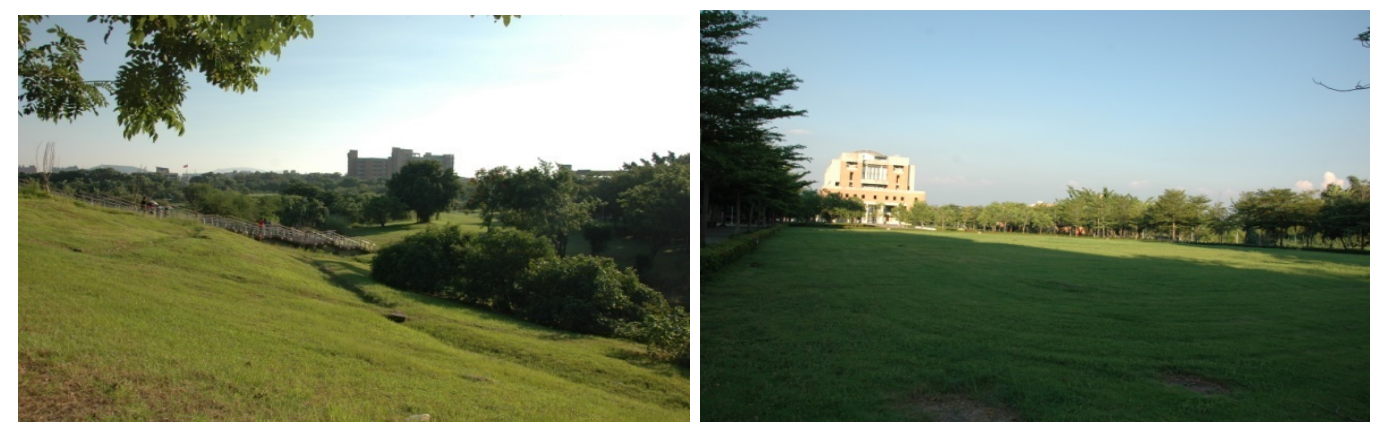

Fig. 6 Fig. 7 Green vegetation of the campus

\section{Path planning and careful separation of people and vehicles}

University internal traffic in the non motor vehicle and pedestrian as a starting point to consider the issue, only from the necessary vehicle transportation, logistics, such as bus, the car use perspective. Many university in order to reduce the interference to the teaching to take various measures to limit vehicle access and speed. Another feature is the presence of a large university traffic flow of traffic, a large number of people during the schools out, rationality is the test of campus planning. People walk, talk, often accompanied by thinking, waiting for the rest and other activities, so that it must be the separation of people and vehicles design. It must be the separation of people and vehicles design. Only take full account of these characteristics, can be designed to meet the requirements of the traffic transportation system of university.

Design of Kaohsiung First University of science and technology of the campus to make full use of the separation of people and vehicles. Which including the plane and three-dimensional shunt. In the campus the main road, the plane distribution, a clear distinction between the pavement, road vehicles and non motorized vehicles, motor vehicle and non motor vehicle flow separated flow. And in the building of the students living area, car stereo diversion plan. By this way, the vehicles and pedestrians from different directions into the area, to avoid mutual interference and cross.



Fig. 8 Car and plane road diversion primary school flow of the car and student

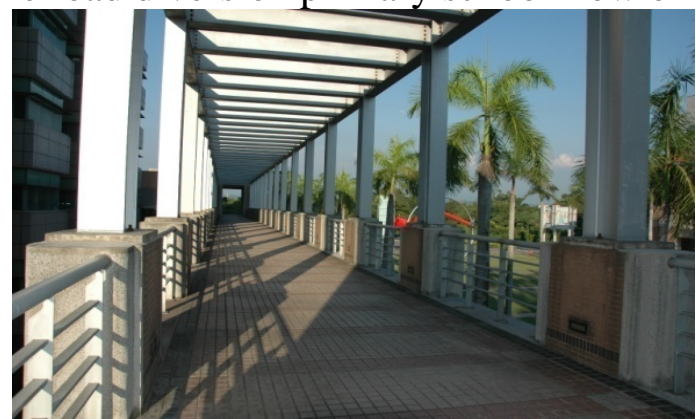

Fig. 9 use the corridor to realize the flow of the car and student

\section{Attaching importance to campus culture humanities landscape}

There are many university campus with a memorial or landmark and sketch. These elements in the process of university development, has gradually become a unique campus landscape, giving the campus with rich historical and cultural connotation, its symbolic significance is far more than the 
use of function. These "campus landmark" in the apparent position of the campus, is an important element, environmental organizations, and symbolic significance. Campus landmarks in the form of no fixed pattern, scale, the scale is big or small, is a small building, or even a sculpture, are likely to become the epitome of campus spirit, its role can not be ignored

Kaohsiung First University of science and technology, in order to highlight the culture of the school, to spend in the landscape design of the campus thoughts. In the campus, more than a dozen sculptures placed, including "fun puzzle", "water", "landscape", "a stone of hope." notes and so on, the more well-known period, and as a symbol of the school publicity device works "360 degrees". From Taiwan modern sculptor Ding Shuiquan, Chen Yizhang as the prototype for the creation of a complete, harmonious, symbol, a rigorous, professional, the meaning of the standard.

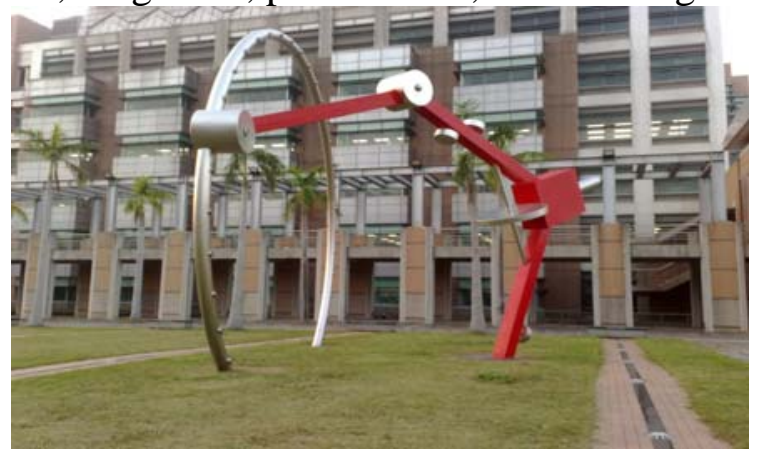

Fig. 10 Sculpture "360 degrees"

\section{Conclusion}

With the change of the connotation of modern university education, not just the narrow technical or occupation education training, but pay more attention to comprehensive ability, so that students in the complicated and changeable social life, social networking, with access to knowledge and practical ability of innovation. Therefore in the design of campus planning, to fully consider the user's status, combined with the actual situation, design a reasonable distribution of the building. Campus planning as a science, it is related to the school is responsible for the project, responsible for staff and students and future perspective, the campus planning and construction in Colleges and universities must be careful and scientific.

The scientific planning of the campus can give teachers and students to provide a good environment, more conducive to the cultivation of talents, but also can alleviate the pressure of high load research work, at the same time inspiration, influence character by environment influence on students' character, shaping positive values. Say from this aspect, Kaohsiung First University of science and technology has made a very good example of.

\section{Reference:}

[1]Wu Lina. The university campus environment and the creation of beauty, Tsinghua University, [D]. 1989.

[2]ShenGuoyao. Discussion on university campus environment planning theory. Journal of Architecture [J]. 1991.

[3]Song Zefang, Zhou Yihu. The university campus planning and architectural design. [M] .China Building Industry Press. 2006.

[4]Zhao Jialin. Time and space of campus planning. [M]. Garden City Publishing House. 1998.

[5]Peng Kangjian. Study on the university campus space model [D]. Architectural design and Research Institute of Tokai University.1985. 